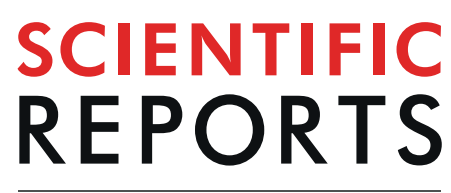

natureresearch

\title{
Sensing mechanism of a ratiometric near-infrared fluorescent chemosensor for cysteine hydropersulfide: Intramolecular charge transfer
}

\author{
Xiaofei Sun ${ }^{1}$, Aihua Gao ${ }^{2} \&$ Hongxing Zhang ${ }^{1 *}$ \\ Previous studies have shown that the cysteine hydropersulfide (Cys-SSH) as the sulfur donor is crucial \\ to sulfur-containing cofactors synthesis. Recently, a selective and sensitive near-infrared ratiometric \\ fluorescent chemosensor Cy-DiSe has been designed and synthesized to detect Cys-SSH spontaneously. \\ Herein, by means of the density functional theory (DFT) and time-dependent density functional \\ theory (TD-DFT) approaches, the sensing mechanism has been thoroughly explored. According to our \\ calculations, the experimental data have been reproduced. The results indicate the intramolecular \\ charge transfer (ICT) is the reason for changes in fluorescence wavelengths. Compared with the \\ chemosensor Cy-DiSe, the larger energy gap of Cy induced by ICT mechanism leads to the blue-shift \\ of the absorption and emission spectra, which guarantees that $\mathrm{Cy}$-DiSe can become a ratiometric \\ fluorescent chemosensor to detect Cys-SSH.
}

As the widespread sulfur-containing biomolecules, reactive sulfur species (RSS) are essential in signal transduction and antioxidant physiological processes ${ }^{1,2}$. Among which, cysteine hydropersulfide (Cys-SSH), as the main source of hydropersulfide derivatives, is crucial to the synthesis of sulfur-containing cofactors, activating or inactivating enzyme activities, modulating cellular signaling, and regulating the cellular redox equilibrium ${ }^{3-6}$. Thus, it is important to obtain the concentration of intracellular Cys-SSH in real-time and in situ.

Compared with other biological detection technologies, fluorescent chemosensors have been exploited as the indispensable technique for detecting a variety of intracellular reactive species, due to their apparent advantages such as less invasiveness, operational simplicity, high sensitivity and selectivity ${ }^{7-20}$. Among these studies, ratiometric fluorescent chemosensors have attracted enormous attentions because they are able to function regardless of the external interferences, such as the concentration and polarity of solution, optic pollution and biological auto-fluorescence interference. Up to now, a mass of ratiometric fluorescent chemosensors have been synthesized by chemists ${ }^{16,17,21-28}$. It has been demonstrated that they can quantitatively detect bioactive molecules both in vitro and in $v i v o^{17,24,25,28-30}$. To the best of our knowledge, attentions are mainly focused on the novel synthesis of fluorescent chemosensors, and most of the sensing mechanisms are based on speculation ${ }^{17,24,25,28-30}$. Nevertheless, explorations on the sensing mechanisms of fluorescent chemosensors are also essential to design more effective fluorescent chemosensors. More importantly, with the help of computational chemistry, the relevant photophysical process and detailed sensing mechanism for fluorescent chemosensors can be comprehensively investigated.

Until now, numerous sensing mechanisms have been proposed and elucidated for designing fluorescent chemosensors, including the intramolecular charge transfer (ICT) ${ }^{17,22,30-36}$, the photoinduced electron transfer $(\mathrm{PET})^{8,9,37-40}$, the excited state proton transfer $(\mathrm{ESPT})^{16,41-45}$, and the fluorescence resonance energy transfer $(\text { FRET })^{46-50}$. Thereinto, ICT mechanism is usually adopted to design the ratiometric fluorescent chemosensors $^{17,21,22,29,34,51,52}$. An ICT-based ratiometric fluorescent chemosensor can lead to the fluorescence spectrum displacement by enhancing or suppressing such an ICT process accompanying with partial charge transfer ${ }^{7,32,53}$.

${ }^{1}$ Laboratory of Theoretical and Computational Chemistry, Institute of Theoretical Chemistry, Jilin University, Changchun, 130023, China. 'School of Physics and Optoelectronic Engineering, Ludong University, Yantai, 264025, China. *email: zhanghx@jlu.edu.cn 


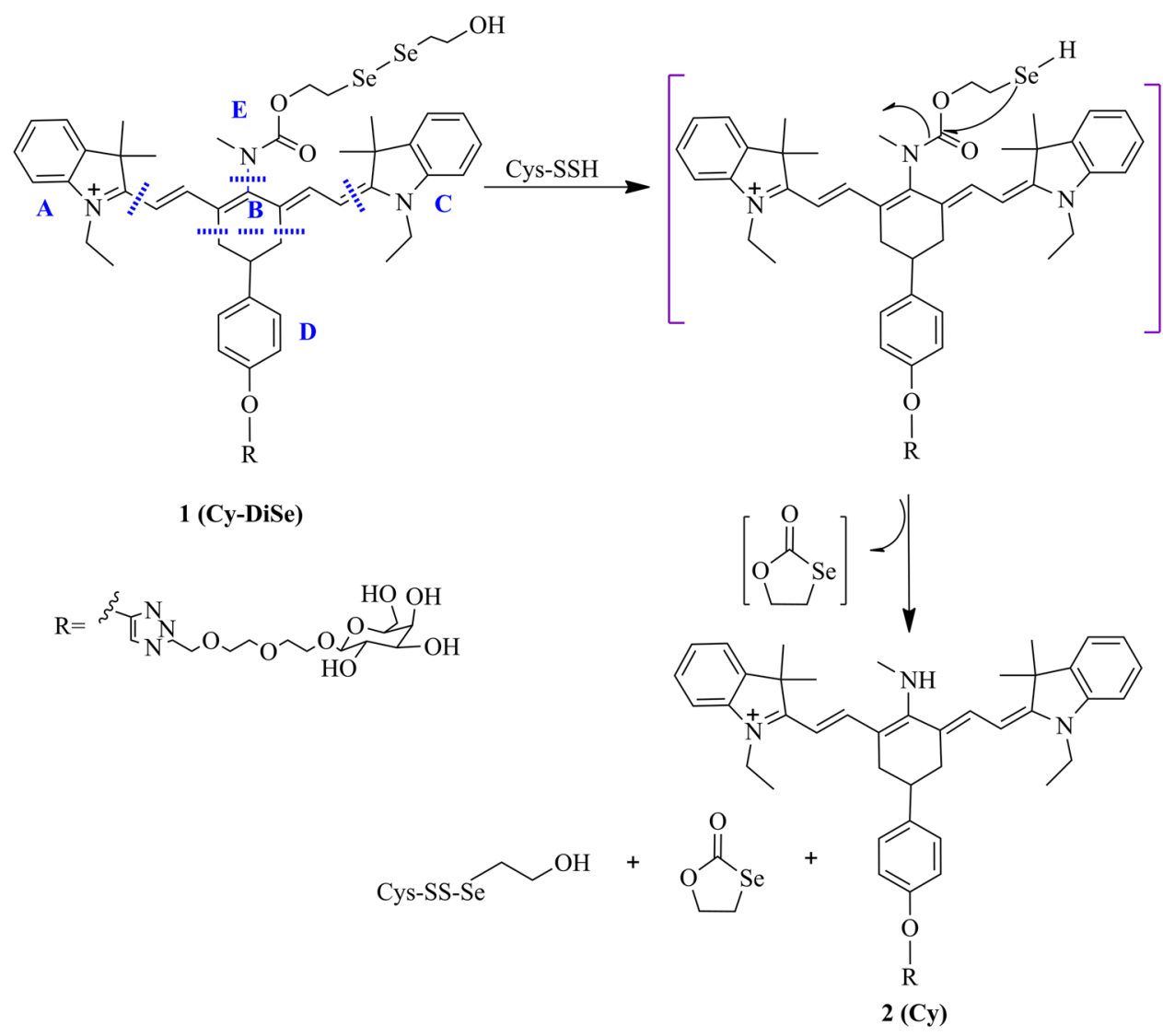

Figure 1. Proposed reaction mechanism for Cys-SSH detection.

Recently, Han et al. ${ }^{17}$. developed a near-infrared (NIR) ratiometric fluorescent chemosensor Cy-DiSe, which was designed according to the ICT mechanism, to detect Cys-SSH. Upon the detection of Cys-SSH, obvious changes in the spectra can be observed. The maximum value of absorption spectrum changes from $790 \mathrm{~nm}$ to $614 \mathrm{~nm}$, while the maximum emission spectrum exhibits blue-shift from $797 \mathrm{~nm}$ to $749 \mathrm{~nm}$. As shown in Fig. 1, after adding Cys-SSH to Cy-DiSe, a two-step reaction process occurs. Based on the reduction of diselenide, the intermediate is formed instantly, then through the intramolecular cyclization, the five-membered cyclic selenocarbonate is removed and the elimination product $\mathrm{Cy}$ is obtained ultimately. The authors only propose the reaction mechanism of modulating fluorescence changes, while the sensing mechanism still needs to be further clarified.

To elucidate the concrete sensing mechanism, we have carried out calculations based on the density functional theory (DFT) and time-dependent density functional theory (TD-DFT) approaches. We investigate the absorption and emission properties of Cy-DiSe and Cy involved in the sensing process. First, the ground $\left(\mathrm{S}_{0}\right)$ and excited $\left(\mathrm{S}_{1}\right)$ state geometries of the two molecules are optimized. Then, based on the optimized structures, the electronic transition energies, oscillator strengths, as well as the frontier molecular orbitals (FMOs) are analyzed. Besides, the $\mathrm{D}_{\mathrm{CT}}$ and $\Delta \mathrm{r}$ indexes ${ }^{54-56}$, which can be adopted to measure the charge-transfer length and the hole-electron distance, are also provided to verify the ICT quantitatively. Our theoretical calculations not only reveal the ICT sensing mechanism, but also illustrate the reason why Cy-DiSe can serve as a ratiometric near-infrared fluorescent chemosensor for Cys-SSH.

\section{Results and Discussion}

Optimized geometries. The galactose group in Cy-DiSe enables it to target the liver, while it is speculated not to influence the relevant fluorescent properties. Therefore, the galactose group is replaced by methyl group for the sake of simplification. By adjusting the position of two indole rings in the cyanine skeleton, three optimized structures of chemosensor Cy-DiSe, namely, a, a1 and a2 (shown in Fig. S1), are obtained at the ground state. The energy of a is $0.10 \mathrm{eV}$ and $0.19 \mathrm{eV}$ lower than that of a1 and a2, and a is chosen as the most stable configuration for the following research. The optimized ground and excited state geometries of the Cy-DiSe and its elimination product $\mathrm{Cy}$ are displayed in Fig. 2. The atomic coordinates of these structures are provided in the electronic supplementary information (ESI). In the ground state, the calculated $\mathrm{C}_{2}-\mathrm{N}_{3}$ bond length and $\mathrm{C}_{1}-\mathrm{C}_{2}-\mathrm{N}_{3}-\mathrm{C}_{4}$ dihedral angle of Cy-DiSe are $1.44 \AA$ and $90.9^{\circ}$, respectively. While in the excited state, the corresponding values are $1.44 \AA$ and $89.8^{\circ}$, which are similar to the ground state. There is no obvious difference in geometries between the ground and excited states for Cy-DiSe. However, a noticeable change in the steric configuration of Cy has been observed. The bond length of $\mathrm{C}_{2},-\mathrm{N}_{3}$, is $1.36 \AA$ in the ground state, which is lengthened to $1.41 \AA$ in the excited 

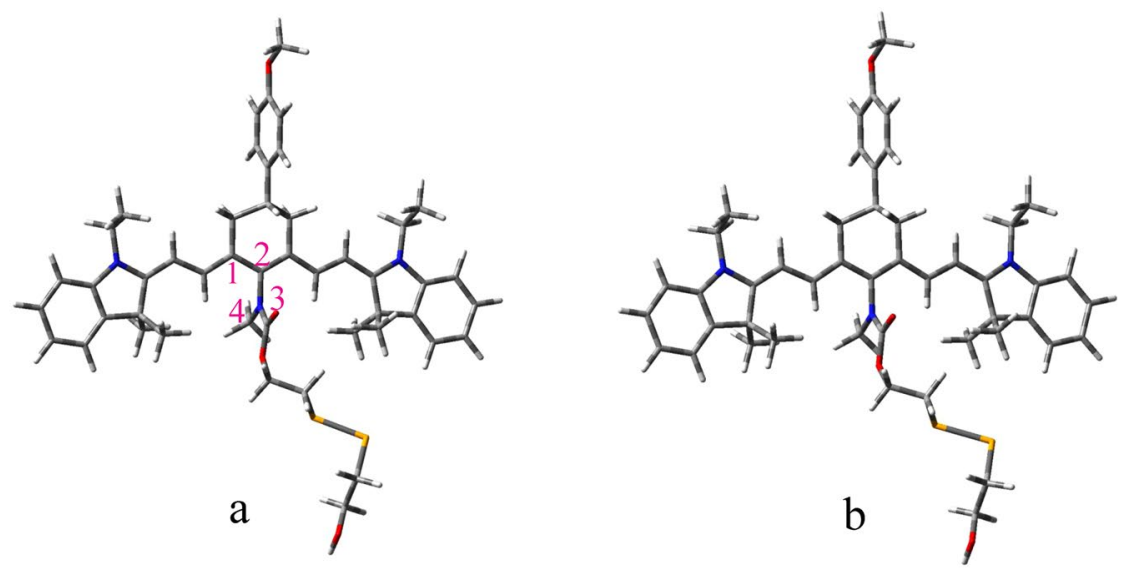

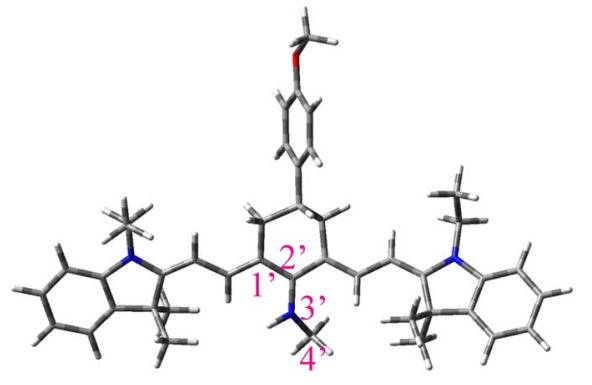

c

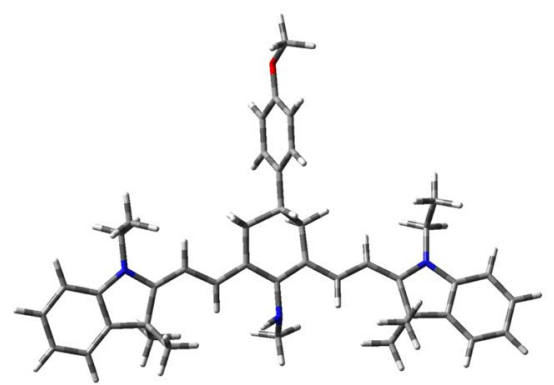

d

Figure 2. Optimized structures of Cy-DiSe and Cy in the ground (a,c) and excited (b,d) states, respectively.

\begin{tabular}{|l|l|l|l|l|l|l|}
\hline Compound & $\begin{array}{l}\text { Electronic } \\
\text { transition }\end{array}$ & $\begin{array}{l}\text { Energy } \\
(\mathbf{n m} / \mathbf{e V})\end{array}$ & $\begin{array}{l}\text { Experimental } \\
\text { absorption }(\mathbf{n m} / \mathbf{e V})\end{array}$ & $\boldsymbol{f}$ & Composition & CI \\
\hline Cy-DiSe & $\mathrm{S}_{0} \rightarrow \mathrm{S}_{1}$ & $630 / 1.97$ & $790 / 1.57$ & 2.2938 & HOMO $\rightarrow$ LUMO & $98.2 \%$ \\
\hline $\mathrm{Cy}$ & $\mathrm{S}_{0} \rightarrow \mathrm{S}_{1}$ & $566 / 2.19$ & $614 / 2.02$ & 2.0955 & HOMO $\rightarrow$ LUMO & $99.9 \%$ \\
\hline
\end{tabular}

Table 1. Comparison of experimental and calculated absorption at the TD-DFT/B3LYP/6-311 G(d) level.

state. Simultaneously, Cy exhibits significant conformational rotation with the dihedral angle of $\mathrm{C}_{1},-\mathrm{C}_{2},-\mathrm{N}_{3},-\mathrm{C}_{4}$, decreasing from $143.9^{\circ}$ to $105.6^{\circ}$ upon excitation.

Absorption property analysis. Based on the optimized ground state structures, the low-lying singlet electronic transition energies and the corresponding oscillator strengths as well as the transition compositions of Cy-DiSe and Cy have been calculated and summarized in Table 1. In the experiment ${ }^{17}$, the initial absorption peak of Cy-DiSe lies in $790 \mathrm{~nm}$. The addition of Cys-SSH results in a significant blue-shift, that is, with the increase of Cys-SSH concentration, the absorption peak at $790 \mathrm{~nm}$ gradually decreases in its height, while an increase of the new absorption peak at $614 \mathrm{~nm}$ is observed. The lowest electronic transition energies of Cy-DiSe and Cy have been calculated and shown in Table 1. Among all the calculated results, only the absorption spectrum of Cy-DiSe fails to be accurately reproduced because of the strong overestimation on the lowest excitation energy, regardless numerous methods have been tested (shown in Table S2). This result, though exhibiting difference comparing to experiment, is consistent with numbers of previous theoretical studies about cyanine ${ }^{57-61}$. The systematic and large overestimation of the lowest singlet excitation energy has been attributed to the difficulty of capturing the differential electron-correlation effects between ground and excited states, no matter what functionals, basis sets, or ground state geometries ${ }^{57-61}$ have been chosen. In fact, it is one of the theoretical signatures of cyanine that the obtained transition energy is rather insensitive to the selection of a specific (pure or hybrid) exchange-correlation functional ${ }^{59,60}$. It should also to note that, although the lowest singlet excitation energy is overestimated, the geometries and vibrational signatures in this study are accurate ${ }^{60,61}$. For Cy-DiSe, the first singlet-transition $\left(\mathrm{S}_{0} \rightarrow \mathrm{S}_{1}\right)$ is the dominant transition with the largest oscillator strength of 2.2938 , which is mainly assigned to the highest occupied molecular orbital (HOMO) $\rightarrow$ the highest occupied molecular orbital (LUMO). Similarly, the calculated results reveal that an intense $S_{0} \rightarrow S_{1}$ excitation with the largest oscillator strength of 2.0955 as the primary transition corresponds to $\mathrm{HOMO} \rightarrow$ LUMO for Cy.

Figure 3 displays the calculated frontier molecular orbitals for Cy-DiSe and Cy, so as to reveal the blue-shift of the emitted fluorescence after adding Cys-SSH. The MO distributions in HOMO for the two molecules are 


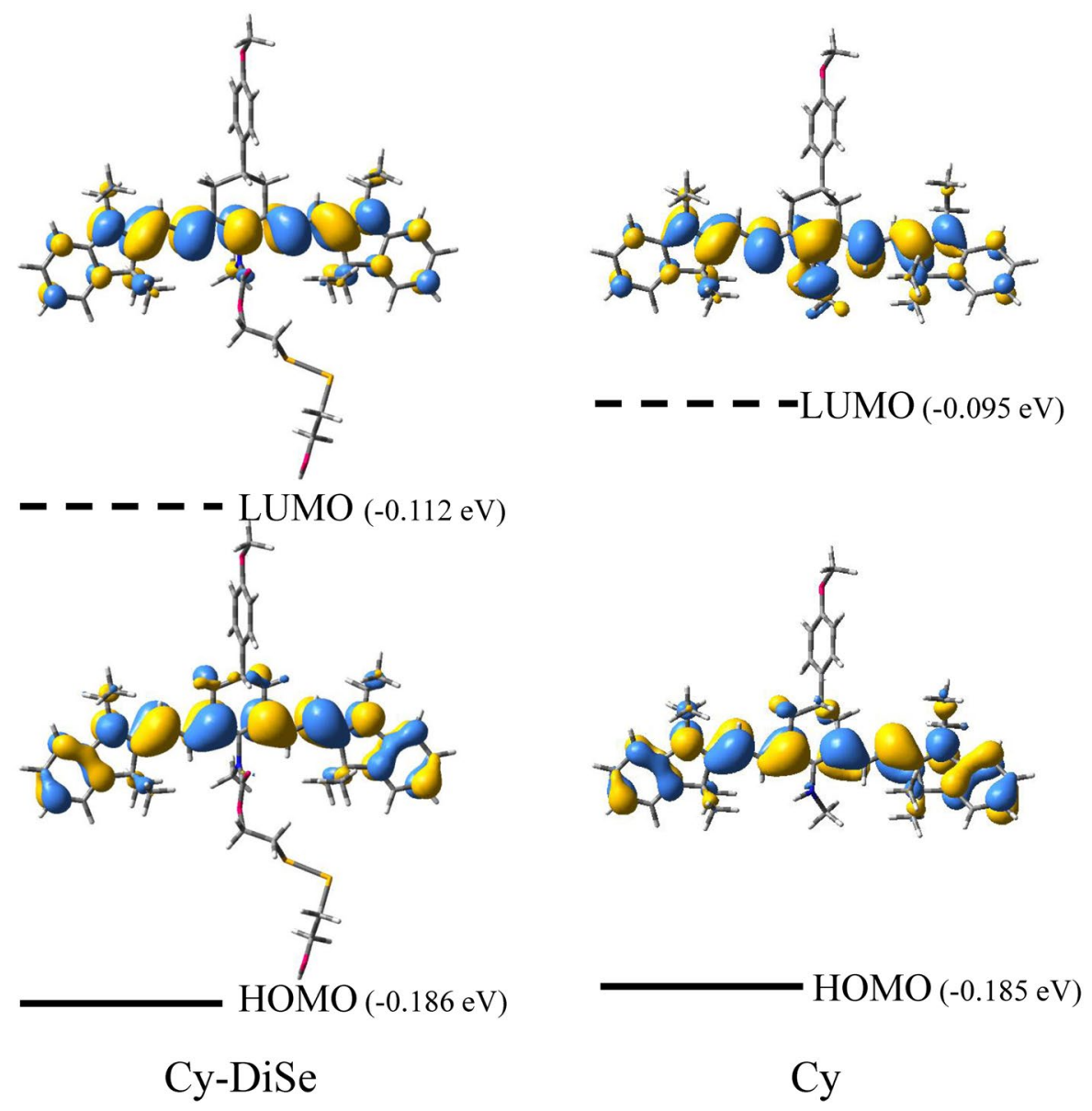

Figure 3. Calculated frontier molecular orbitals HOMO and LUMO in the absorption of Cy-DiSe and Cy, respectively.

\begin{tabular}{|l|l|l|l|l|l|l|}
\hline \multirow{3}{*}{ Compound } & \multirow{6}{|l|}{ Composition (\%) } \\
\cline { 3 - 8 } & MO & A & B & C & D & E \\
\hline \multirow{2}{*}{ Cy-DiSe } & HOMO & 22.436 & 49.340 & 22.170 & 5.425 & 0.629 \\
\cline { 2 - 7 } & LUMO & 23.433 & 49.555 & 23.620 & 0.804 & 2.588 \\
\hline \multirow{2}{*}{ Cy } & HOMO & 26.360 & 46.248 & 21.715 & 4.901 & 0.776 \\
\cline { 2 - 7 } & LUMO & 19.083 & 48.203 & 21.367 & 1.379 & 9.969 \\
\hline
\end{tabular}

Table 2. Molecular orbital compositions in $\mathrm{S}_{0}$ state geometries.

delocalized over the conjugated chain of cyanine, while the LUMO for both molecules share the similar distribution with that of HOMO. At the same time, the increase of the MO distribution on group E (see Fig. 1) accompanying with $\mathrm{HOMO} \rightarrow$ LUMO transition implies that the $\mathrm{S}_{1}$ state of Cy own the ICT character. Figure 3 shows that the elimination of diselenide group leads to an increase of LUMO energy of Cy. Therefore, the larger energy gap of Cy induces the spectral blue-shift in contrast with Cy-DiSe. Such large obvious blue-shift between the spectra of Cy-DiSe and its elimination product $\mathrm{Cy}$ is essential for $\mathrm{Cy}$-DiSe to act as a ratiometric fluorescent chemosensor for Cys-SSH.

Molecular orbital compositions and indexes. In order to quantitatively describe the ICT character, atoms in both Cy-DiSe and Cy are categorized into five parts (A, B, C, D and E), respectively, among which the cyanine skeleton is subdivided into parts $\mathrm{A}, \mathrm{B}$ and $\mathrm{C}$ (shown in Fig. 1). The contribution of each part to the FMOs is calculated based on the ground state and the corresponding results are listed in Table 2. Neither Cy-DiSe nor Cy exhibits charge transfer between HOMO and LUMO on parts B and C. However, compared with Cy-DiSe, an obvious charge transfer occurs on one of the indole ring A from cyanine skeleton of Cy. An electron transition is observed from $26.360 \%$ on HOMO to $19.083 \%$ on LUMO. Besides, for part D of Cy-DiSe and Cy, the MO distribution decreases about 3\% from HOMO to LUMO. More importantly, part E of Cy exhibits obvious charge transfer compared to Cy-Dise with a MO distribution difference at around 9\% between HOMO and LUMO, while 


\begin{tabular}{|l|l|l|l|}
\hline Compound & $\boldsymbol{\Delta} \mathbf{r}(\AA)$ & $\mathbf{D}_{\text {CT }}(\AA)$ & $\mathbf{q}_{\text {CT }}\left(\left|\mathbf{e}^{-}\right|\right)$ \\
\hline Cy-DiSe & 0.45 & 0.57 & 0.40 \\
\hline Cy & 0.80 & 0.89 & 0.48 \\
\hline
\end{tabular}

Table 3. Computed hole-electron distance, CT length and transferred charge for the S1 states of Cy-DiSe and Cy.

\begin{tabular}{|l|l|l|l|l|l|l|}
\hline Compound & $\begin{array}{l}\text { Electronic } \\
\text { transition }\end{array}$ & $\begin{array}{l}\text { Energy } \\
(\mathbf{n m} / \mathbf{e V})\end{array}$ & $\begin{array}{l}\text { Experimental } \\
\text { emission }(\mathbf{n m} / \mathbf{e V})\end{array}$ & $\boldsymbol{f}$ & Composition & CI \\
\hline Cy-DiSe & $\mathrm{S}_{1} \rightarrow \mathrm{S}_{0}$ & $772 / 1.61$ & $797 / 1.56$ & 2.6054 & LUMO $\rightarrow$ HOMO & $99.6 \%$ \\
\hline $\mathrm{Cy}$ & $\mathrm{S}_{1} \rightarrow \mathrm{S}_{0}$ & $725 / 1.71$ & $749 / 1.66$ & 2.4889 & LUMO $\rightarrow$ HOMO & $99.4 \%$ \\
\hline
\end{tabular}

Table 4. Comparison of experimental and calculated emission at the TD-DFT/B3LYP/6-311 G(d) level.

in condition of Cy-DiSe, only about $2 \%$ is increased. From the results above, one can conclude that partial charge transfer from cyanine skeleton to methyl amino group exists in Cy upon excitation. Therefore, the $\mathrm{S}_{1}$ state of Cy shows the relatively distinct ICT character.

In addition, a quantitative analysis is performed to measure the hole-electron distance and the charge-transfer length by calculating the two indexes, namely $\Delta \mathrm{r}$ and $\mathrm{D}_{\mathrm{CT}}{ }^{56}$. The calculated results of $\Delta \mathrm{r}$ and $\mathrm{D}_{\mathrm{CT}}$ for Cy-DiSe and Cy are listed in Table 3, accompanying with the transferred charge $\left(\mathrm{q}_{\mathrm{CT}}\right)$ between the donor and acceptor groups. The values of $\Delta \mathrm{r}$ and $\mathrm{D}_{\mathrm{CT}}$ are larger, the characteristic of charge separation is more apparent, that is, the ICT character is more significant. For Cy-DiSe, the small values of $\Delta \mathrm{r}$ and $\mathrm{D}_{\mathrm{CT}}$ (about $0.5 \AA$ ) imply that there is almost no ICT character. Compared with Cy-DiSe, Cy exhibits the relatively obvious ICT character, which can be verified by the larger values close to $1 \AA$ of $\Delta \mathrm{r}$ and $\mathrm{D}_{\mathrm{CT}}$.

Emission property and sensing mechanism. According to the viewpoint of analytical science, the fluorescent emission of a chemosensor is more sensitive for detection than the corresponding UV-Vis absorption ${ }^{62}$. Thus, the fluorescent characters of $\mathrm{Cy}$-DiSe and its elimination product $\mathrm{Cy}$ are investigated to verify the potential of Cy-DiSe as a ratiometric fluorescent chemosensor for Cys-SSH. The sensing mechanism can thus be understood better. According to our calculations, all the involved excited states are the first singlet excited states. The optimized $\mathrm{S}_{1}$ state geometries of Cy-DiSe and Cy are displayed in Fig. 2, and the corresponding atomic coordinates are supplied in the ESI. Then, the emission properties of the two molecules are investigated using the TD-DFT method based on the optimized excited state structures. Table 4 shows their low-lying singlet electronic transition energies, oscillator strengths as well as the transition compositions and the relevant FMOs for the emission are given in Fig. 4.

As shown in Fig. 4 and Table 4, the calculated emission wavelengths of Cy-DiSe and Cy are in agreement with the experimental measurements. For Cy-DiSe, the calculated emission wavelength is $772 \mathrm{~nm}$ and the experimental result lies in $797 \mathrm{~nm}$. With the addition of Cys-SSH, the wavelength in experiment blue-shifts to $749 \mathrm{~nm}$, while the calculated emission wavelength of Cy is $725 \mathrm{~nm}$. Besides, for both experimental and calculated results, the emission spectrum of $C y$ all shows a blue-shift of about $50 \mathrm{~nm}$ compared with Cy-DiSe, which ensures that Cy-DiSe can act as the ratiometric fluorescent chemosensor. As can be seen from Table 4 and Fig. 4, the main relaxation transition for Cy-DiSe and Cy is $S_{1} \rightarrow S_{0}$ transition assigned to LUMO $\rightarrow$ HOMO with the largest oscillator strengths. Thus, the $S_{1}$ state is a bright state which results in the strong fluorescence. Meanwhile, accompanying with LUMO $\rightarrow$ HOMO transition, the charge transfer between the nitrogen group and the cyanine skeleton implies that the $S_{1}$ state of Cy exhibits the ICT character. The larger energy gap of Cy leads to the emission spectrum blue-shift compared with Cy-DiSe. Based on the calculated emission results, Cy-DiSe is confirmed again that it can serve as a ratiometric fluorescent chemosensor on detecting Cys-SSH.

According to the above calculated results, the sensing mechanism of the chemosensor Cy-DiSe can be depicted as follows. The added Cys-SSH triggers the $\mathrm{O}=\mathrm{C}-\mathrm{N}$ single bond cleavage reaction in Cy-DiSe and its elimination product $\mathrm{Cy}$ is obtained subsequently, which leads to the change of the electron density distribution. The locally excited state is responsible for the strong fluorescence emission, at the same time, the $S_{1}$ state of Cy possesses ICT between the cyanine skeleton and nitrogen group. The larger energy gap of Cy results in the spectrum blue-shift compared with Cy-DiSe. Thus, Cy-Dise can serve as an excellent candidate of ratiometric fluorescent chemosensor when the ICT process is triggered by Cys-SSH.

\section{Conclusions}

In summary, DFT and TDDFT methods have been applied to investigate the sensing mechanisms of the ratiometric fluorescent chemosensor Cy-DiSe and its elimination product $\mathrm{Cy}$. The optimized geometries of the two molecules are obtained and there are no obvious differences between the ground and the excited states. It has been demonstrated that the theoretical calculated spectrum blue-shift, the strong fluorescence emission, and the large Stokes shift are all coincided with the experimental measurements. As the dominant transition for Cy-DiSe and $\mathrm{Cy}, \mathrm{S}_{0} \rightarrow \mathrm{S}_{1}$ is assigned to $\mathrm{HOMO} \rightarrow$ LUMO with the electron density mainly localized on the conjugated cyanine chain, which results in the strong fluorescence emission. Meanwhile, the partial intramolecular charge transfer from A and D groups of cyanine skeleton to the amino contained group E leads to the $S_{1}$ state of Cy exhibit the ICT character. The calculated contribution results of each part to the molecular orbitals and the $\Delta \mathrm{r}$ and $\mathrm{D}_{\mathrm{CT}}$ indexes provide further evidences that the $\mathrm{S}_{1}$ state of Cy possesses the ICT character. Because of the 


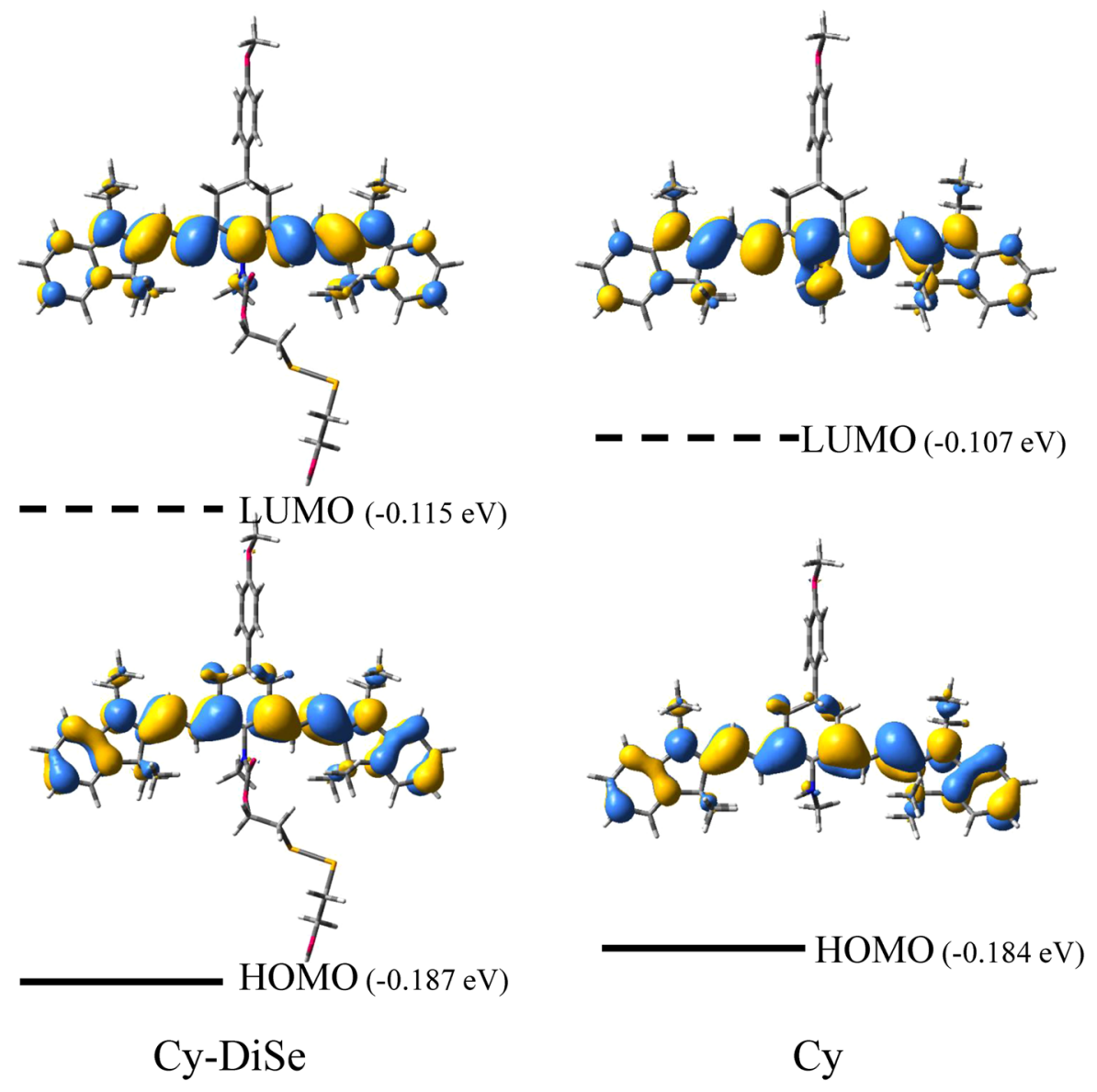

Figure 4. Calculated frontier molecular orbitals HOMO and LUMO in the emission of Cy-DiSe and Cy, respectively.

strong electron donating ability, amino can increase the LUMO energy of Cy, thus, the larger energy gap leads to the absorption spectrum blue-shift compared with Cy-DiSe. Accordingly, the calculated emission property results of $\mathrm{Cy}$-DiSe and Cy are similar to those of absorption property. The large observable spectrum blue-shift supports that Cy-DiSe has the potential as a ratiometric fluorescent chemosensor when the ICT process is triggered by Cys-SSH. Our calculated results are helpful for the design of new ratiometric chemosensors with novel fluorescent properties based on the ICT sensing mechanism in future.

\section{Methods}

In the present work, all theoretical calculations are carried out by using the Gaussian 09 program package ${ }^{63}$. The ground and first excited state geometries are optimized using the DFT/TD-DFT methods, respectively. Afterwards, the absorption and emission properties are further investigated by the TD-DFT methods. All geometric optimization calculations are completed without constrains for symmetry, bonds, angles or dihedral angles. The vibrational frequency analyses are carried out to ensure that each optimized structure is the real minimum without imaginary vibration frequency. In order to find the appropriate functional and basis set, a series of different functionals and basis sets have been tested shown in Table S2. Taking into account of the accuracy and computation efficiency, the Becke's three-parameter hybrid exchange functional with Lee-Yang-Parr gradient-corrected correlation (B3LYP functional) ${ }^{64-67}$ and the $6-311 \mathrm{G}(\mathrm{d})^{68,69}$ are selected for the following theoretical calculations. In all calculations, the solvent effect is included using the conductor-like polarizable continuum model (CPCM) with the dielectric constant of water $(\varepsilon=80.1)$ and the solute cavity is built with a special set of atomic radii as sug gested by Klamt ${ }^{70,71}$. Molecular orbital compositions and the indexes of $\Delta \mathrm{r}$ and $\mathrm{D}_{\mathrm{CT}}$, which are used to indicate the hole-electron distance and the charge-transfer length, are calculated using the Multiwfn program $^{72}$ to describe the ICT character quantitatively.

Received: 30 July 2019; Accepted: 21 December 2019;

Published online: 20 January 2020

\section{References}

1. Paul, B. D. \& Snyder, S. H. $\mathrm{H}_{2}$ S signalling through protein sulfhydration and beyond. Nat. Rev. Mol. Cell Biol. 13, 499-507 (2012).

2. Wallace, J. L. \& Wang, R. Hydrogen sulfide-based therapeutics: Exploiting a unique but ubiquitous gasotransmitter. Nat. Rev. Drug Discov. 14, 329-345 (2015).

3. Toohey, J. I. Sulfur signaling: Is the agent sulfide or sulfane? Anal. Biochem. 413, 1-7 (2011). 
4. Luebke, J. L. et al. The CsoR-like sulfurtransferase repressor (CstR) is a persulfide sensor in Staphylococcus aureus. Mol. Microbiol. 94, 1343-1360 (2014).

5. Ida, T. et al. Reactive cysteine persulfides and S-polythiolation regulate oxidative stress and redox signaling. Proc. Natl. Acad. Sci. USA 111, 7606-7611 (2014).

6. Kimura, Y. et al. 3-Mercaptopyruvate sulfurtransferase produces potential redox regulators cysteineand glutathione-persulfide (CysSSH and GSSH) together with signaling molecules $\mathrm{H}_{2} \mathrm{~S}_{2}, \mathrm{H}_{2} \mathrm{~S}_{3}$ and $\mathrm{H}_{2} \mathrm{~S}$. Sci. Rep. 7, 10459 (2017).

7. Silva, A. P. D. et al. Signaling recognition events with fluorescent sensors and switches. Chem. Rev. 97, 1515-1566 (1997).

8. Miura, T. et al. Rational design principle for modulating fluorescence properties of fluorescein-based probes by photoinduced electron transfer. J. Am. Chem. Soc. 125, 8666-8671 (2003).

9. Han, F. et al. 3,6-Disubstituted carbazole-based bisboronic acids with unusual fluorescence transduction as enantioselective fluorescent chemosensors for tartaric acid. J. Org. Chem. 74, 1333-1336 (2009).

10. Wu, J. S., Liu, W. M., Ge, J. C., Zhang, H. Y. \& Wang, P. F. New sensing mechanisms for design of fluorescent chemosensors emerging in recent years. Chem. Soc. Rev. 40, 3483-3495 (2011).

11. Pollock, J. B., Cook, T. R. \& Stang, P. J. Photophysical and computational investigations of bis(phosphine) organoplatinum(II) metallacycles. J. Am. Chem. Soc. 134, 10607-10620 (2012).

12. Wang, B. S. et al. A reversible fluorescence probe based on Se-BODIPY for the redox cycle between $\mathrm{HClO}$ oxidative stress and $\mathrm{H}_{2} \mathrm{~S}$ repair in living cells. Chem. Commun. 49, 1014-1016 (2013).

13. Qu, Z. J., Ding, J. X., Zhao, M. Y. \& Li, P. Development of a selenide-based fluorescent probe for imaging hypochlorous acid in lysosomes. J. Photoch. Photobio. A 299, 1-8 (2015).

14. Wang, J. M., Yu, H., Li, Q. \& Shao, S. J. A BODIPY-based turn-on fluorescent probe for the selective detection of hydrogen sulfide in solution and in cells. Talanta 144, 763-768 (2015).

15. Chen, Z. J. et al. The N-B interaction through a water bridge: Understanding the chemoselectivity of a fluorescent protein based probe for peroxynitrite. J. Am. Chem. Soc. 138, 4900-4907 (2016).

16. Wang, Y. et al. A simple and rapid turn on ESIPT fluorescent probe for colorimetric and ratiometric detection of biothiols in living cells. Sci. Rep. 7, 4377 (2017).

17. Han, X. Y., Yu, F. B., Song, X. Y. \& Chen, L. X. Quantification of cysteine hydropersulfide with a ratiometric near-infrared fluorescent probe based on selenium-sulfur exchange reaction. Chem. Sci. 7, 5098-5107 (2016).

18. Kaur, A., Kolanowski, J. L. \& New, E. J. Reversible fluorescent probes for biological redox states. Angew. Chem. Int. Ed. 55, 1602-1613 (2016).

19. Sun, W., Guo, S. G., Hu, C., Fan, J. L. \& Peng, X. J. Recent development of chemosensors based on cyanine platforms. Chem. Rev. 116, 7768-7817 (2016).

20. Li, Y. \& Chu, T. S. DFT/TDDFT study on the sensing mechanism of a fluorescent probe for hydrogen sulfide: Excited state intramolecular proton transfer coupled twisted intramolecular charge transfer. J. Phys. Chem. A 121, 5245-5256 (2017).

21. Resendez, A. \& Malhotra, S. V. Boronic acid appended naphthyl-pyridinium receptors as chemosensors for sugars. Sci. Rep. 9, 6651 (2019).

22. Zhu, B. C. et al. A highly selective colorimetric and ratiometric fluorescent chemodosimeter for imaging fluoride ions in living cells. Chem. Commun. 47, 7098-7100 (2011).

23. Xu, P. F. et al. An ESIPT-based highly selective and sensitive probe for the detection of hydrogen sulfide. Tetrahedron Lett. 56, 4007-4010 (2015).

24. Huang, H., Dong, F. Y. \& Tian, Y. Mitochondria-targeted ratiometric fluorescent nanosensor for simultaneous biosensing and imaging of $\mathrm{O}^{2--}$ and $\mathrm{pH}$ in live cells. Anal. Chem. 88, 12294-12302 (2016).

25. Gong, D. Y. et al. Fast and selective two-stage ratiometric fluorescent probes for imaging of glutathione in living cells. Anal. Chem. 89, 13112-13119 (2017).

26. Mehdi, H. et al. Aggregation-induced emission (AIE) fluorophore exhibits a highly ratiometric fluorescent response to $\mathrm{Zn}^{2+}$ in vitro and in human liver cancer cells. Chem. Eur. J. 23, 13067-13075 (2017).

27. Hu, Q. et al. Colorimetric and ratiometric chemosensor for visual detection of gaseous phosgene based on anthracene carboxyimide membrane. Anal. Chem. 90, 8686-8691 (2018).

28. Chen, X. Z. et al. Ratiometric fluorescent probes with a self-immolative spacer for real-time detection of $\beta$-galactosidase and imaging in living cells. Anal. Chim. Acta 1033, 193-198 (2018).

29. Yu, F. B. et al. An ICT-based strategy to a colorimetric and ratiometric fluorescence probe for hydrogen sulfide in living cells. Chem. Commun. 48, 2852-2854 (2012).

30. Lin, Y. D. et al. Reaction-based colorimetric and ratiometric fluorescence sensor for detection of cyanide in aqueous media. Chem. Asian J. 7, 2864-2871 (2012).

31. Grabowski, Z. R. \& Rotkiewicz, K. Structural changes accompanying intramolecular electron transfer: Focus on twisted intramolecular charge-transfer states and structures. Chem. Rev. 103, 3899-4031 (2003).

32. Chen, J. S., Liu, R. Z., Yang, Y. \& Chu, T. S. Intramolecular charge transfer and sensing mechanism for a colorimetric fluoride sensor based on 1,8-naphthalimide derivatives. Theor. Chem. Acc. 133, 1411 (2014).

33. Li, Y., Chen, J. S. \& Chu, T. S. Sensing mechanism for a fluorescent off-on chemosensor for cyanide anion. J. Lumin. 179, 203-210 (2016).

34. Cheng, Y. Y. et al. Unparalleled ease of access to a library of biheteroaryl fluorophores via oxidative cross-coupling reactions: Discovery of photostable NIR probe for mitochondria. J. Am. Chem. Soc. 138, 4730-4738 (2016).

35. Bhat, H. R. \& Jha, P. C. A theoretical study on anion sensing mechanism of multi-phosphonium triarylboranes: Intramolecular charge transfer and configurational changes. Phys. Chem. Chem. Phys. 19, 14811-14820 (2017).

36. Bhat, H. R. \& Jha, P. C. Selective complexation of cyanide and fluoride ions with ammonium boranes: A theoretical study on sensing mechanism involving intramolecular charge transfer and configurational changes. J. Phys. Chem. A 121, 3757-3767 (2017).

37. Goldberg, J. M., Batjargal, S., Chen, B. S. \& Petersson, E. J. Thioamide quenching of fluorescent probes through photoinduced electron transfer: Mechanistic studies and applications. J. Am. Chem. Soc. 135, 18651-18658 (2013).

38. Pedersen, S. K. et al. Aarhus sensor green: A fluorescent probe for singlet oxygen. J. Org. Chem. 79, 3079-3087 (2014).

39. Udhayakumari, D., Velmathi, S., Boobalan, M. S., Venkatesan, P. \& Wu, S. P. Heterocyclic ring based colorimetric and fluorescent chemosensor for transition metal ions in an aqueous medium. J. Lumin. 158, 484-492 (2015).

40. Sun, X. F. et al. DFT/TD-DFT calculations on the sensing mechanism of a dual response near-infrared fluorescent chemosensor for superoxide anion and hydrogen polysulfides: Photoinduced electron transfer. RSC Adv. 6, 104735-104741 (2016).

41. Hsieh, C. C. et al. Comprehensive studies on an overall proton transfer cycle of the ortho-green fluorescent protein chromophore. J. Am. Chem. Soc. 133, 2932-2943 (2011).

42. Zhao, J. F., Liu, X. Y. \& Zheng, Y. J. Controlling excited state single versus double proton transfer for 2,2'-Bipyridyl-3,3'-diol: Solvent effect. J. Phys. Chem. A 121, 4002-4008 (2017).

43. Yang, D. P. et al. Solvent controlling excited state proton transfer reaction in quinolone/isoquinoline-pyrazole isomer QP-I: A theoretical study. J. Phys. Org. Chem. 31, 3729 (2018).

44. Zhou, P. W. \& Han, K. L. Unraveling the detailed mechanism of excited-state proton transfer. Acc. Chem. Res. 51, 1681-1690 (2018).

45. Tang, Z. et al. Excited-state proton transfer mechanism of 2,6-diazaindoles. $\left(\mathrm{H}_{2} \mathrm{O}\right)_{\mathrm{n}}(\mathrm{n}=2-4)$ clusters. J. Phys. Chem. B 122, 3988-3995 (2018). 
46. Lee, M. H., Kim, H. J., Yoon, S., Park, N. \& Kim, J. S. Metal ion induced FRET off-on in tren/dansyl-appended rhodamine. Org. Lett. 10, 213-216 (2008).

47. Kar, C., Adhikari, M. D., Ramesh, A. \& Das, G. NIR- and FRET-based sensing of $\mathrm{Cu}^{2+}$ and $\mathrm{S}^{2-}$ in physiological conditions and in live cells. Inorg. Chem. 52, 743-752 (2013).

48. Pal, S. et al. Effect of metal oxidation state on FRET: A Cu(I) silent but selectively $\mathrm{Cu}(\mathrm{II})$ responsive fluorescent reporter and its bioimaging applications. Dalton Trans. 44, 1761-1768 (2015).

49. Manna, A., Sain, D., Guchhait, N. \& Goswami, S. FRET based selective and ratiometric detection of Al(III) with live-cell imaging. New J. Chem. 41, 14266-14271 (2017).

50. Shah, R., Zhou, A. \& Wagner, C. R. Switch-on fluorescent/FRET probes to study human histidine triad nucleotide binding protein 1 (hHint1), a novel target for opioid tolerance and neuropathic pain. Org. Biomol. Chem. 15, 10230-10237 (2017).

51. Cheng, D. D. et al. A ratiometric fluorescent sensor for $\mathrm{Cd}^{2+}$ based on internal charge transfer. Sensors 17, 2517 (2017).

52. Matsumoto, T., Takamine, H., Tanaka, K. \& Chujo, Y. Design of bond-cleavage-induced intramolecular charge transfer emission with dibenzoboroles and their application to ratiometric sensors for discriminating chain lengths of alkanes. Mater. Chem. Front. 1, 2368-2375 (2017).

53. Chen, J. S., Zhou, P. W., Yang, S. Q., Fu, A. P. \& Chu, T. S. Sensing mechanism for a fluoride chemosensor: Invalidity of excited-state proton transfer mechanism. Phys. Chem. Chem. Phys. 15, 16183-16189 (2013).

54. Bahers, T. L., Adamo, C. \& Ciofini, I. A qualitative index of spatial extent in charge-transfer excitations. J. Chem. Theory Comput. 7, 2498-2506 (2011).

55. Guido, C. A., Cortona, P., Mennucci, B. \& Adamo, C. On the metric of charge transfer molecular excitations: A simple chemical descriptor. J. Chem. Theory Comput. 9, 3118-3126 (2013).

56. Zhou, P. W. \& Zhao, L. Accurate description of excited state intramolecular proton transfer that involves zwitterionic state using optimally tuned range-separated time-dependent density functional theory. Int. J. Quantum Chem. 118, 25618 (2018).

57. Moore, B. II. \& Autschbach, J. Longest-wavelength electronic excitations of linear cyanines: The role of electron delocalization and of approximations in time-dependent density functional theory. J. Chem. Theory Comput. 9, 4991-5003 (2013).

58. Zhekova, H. et al. Applications of time dependent and time independent density functional theory to the first $\pi$ to $\pi^{*}$ transition in cyanine dyes. J. Chem. Theory Comput. 10, 3299-3307 (2014).

59. Filatov, M. \& Huix-Rotllant, M. Assessment of density functional theory based $\Delta$ SCF (self-consistent field) and linear response methods for longest wavelength excited states of extended $\pi$-conjugated molecular systems. J. Chem. Phys. 141, 024112 (2014).

60. Guennic, B. L. \& Jacquemin, D. Taking up the cyanine challenge with quantum tools. Acc. Chem. Res. 48, 530-537 (2015).

61. Zhou, P. W. Why the lowest electronic excitations of rhodamines are overestimated by time-dependent density functional theory. Int. J. Quantum Chem. 118, 25780 (2018).

62. Qian, X. H. et al. "Alive" dyes as fluorescent sensors: Fluorophore, mechanism, receptor and images in living cells. Chem. Commun. 46, 6418-6436 (2010).

63. Frisch, M. J. et al. Gaussian 09, Revision D.01, Gaussian, Inc., Wallingford CT, (2016).

64. Lee, C., Yang, W. T. \& Parr, R. G. Development of the Colle-Salvetti correlation-energy formula into a functional of the electron density. Phys. Rev. B 37, 785-789 (1988).

65. Becke, A. D. Density-functional exchange-energy approximation with correct asymptotic behavior. Phys. Rev. A 38, 3098-3100 (1988).

66. Miehlich, B., Savin, A., Stoll, H. \& Preuss, H. Results obtained with the correlation-energy density functionals of Becke and Lee, Yang and Parr. Chem. Phys. Lett. 157, 200-206 (1989).

67. Becke, A. D. Density-functional thermochemistry. III. The role of exact exchange. J. Chem. Phys. 98, 5648-5652 (1993).

68. Petersson, G. A. et al. A complete basis set model chemistry. I. The total energies of closed-shell atoms and hydrides of the first-row elements. J. Chem. Phys. 89, 2193-2218 (1988).

69. Petersson, G. A. \& Allaham, M. A. A complete basis set model chemistry. II. Open-shell systems and the total energies of the firstrow atoms. J. Chem. Phys. 94, 6081-6090 (1991).

70. Klamt, A. \& Schüürmann, G. COSMO: A new approach to dielectric screening in solvents with explicit expressions for the screening energy and its gradient. J. Chem. Soc., Perkin Trans. 2, 799-805 (1993).

71. Eckert, F. \& Klamt, A. Fast solvent screening via quantum chemistry: COSMO-RS approach. AIChE J. 48, 369-385 (2002).

72. Lu, T. \& Chen, F. W. Multiwfn: A multifunctional wavefunction analyzer. J. Comput. Chem. 33, 580-592 (2012).

\section{Acknowledgements}

This work was supported by the National Natural Science Foundation of China (Grant Nos. 21303068, 81300724,

21673092 and 11704171).

\section{Author contributions}

H.X.Z. supervised the project. X.F.S. completed the theoretical calculation and writing work. A.H.G. offered timely technical support in the operation of software. All authors analyzed the data and commented on the paper.

\section{Competing interests}

The authors declare no competing interests.

\section{Additional information}

Supplementary information is available for this paper at https://doi.org/10.1038/s41598-020-57631-5.

Correspondence and requests for materials should be addressed to H.Z.

Reprints and permissions information is available at www.nature.com/reprints.

Publisher's note Springer Nature remains neutral with regard to jurisdictional claims in published maps and institutional affiliations. 
(c) (i) Open Access This article is licensed under a Creative Commons Attribution 4.0 International License, which permits use, sharing, adaptation, distribution and reproduction in any medium or format, as long as you give appropriate credit to the original author(s) and the source, provide a link to the Creative Commons license, and indicate if changes were made. The images or other third party material in this article are included in the article's Creative Commons license, unless indicated otherwise in a credit line to the material. If material is not included in the article's Creative Commons license and your intended use is not permitted by statutory regulation or exceeds the permitted use, you will need to obtain permission directly from the copyright holder. To view a copy of this license, visit http://creativecommons.org/licenses/by/4.0/.

(C) The Author(s) 2020 